\title{
Seasonal Patterns in Syndromic Surveillance Emergency Department Data due to Respiratory Illnesses
}

\author{
Kelly Johnson*, Alecia Alianell and Rachel Radcliffe \\ South Carolina Department of Health and Environmental Control, Columbia, SC, USA
}

\section{Objective}

To assess the temporal patterns of respiratory illnesses in South Carolina (SC) using syndromic surveillance emergency department (ED) data.

\section{Introduction}

Monitoring trends of respiratory illnesses via syndromic surveillance in SC is performed on a daily basis. SC Syndromic Surveillance primarily utilizes emergency department data, and provides situational awareness regarding broad syndrome categories among hospitals in the state. Respiratory illnesses represent a significant public health burden, causing the second highest number of outbreaks reported in SC. Since syndromic surveillance can potentially serve as an earlier indicator of outbreaks, ${ }^{1}$ it is beneficial to assess seasonality of respiratory illnesses to identify illness clusters early to mobilize a rapid response.

\section{Methods}

Near real-time data sent daily from 22 SC hospitals to the state's health department for 2010-2012 were used for this study. The data was encrypted and sent automatically via the Public Health Information Network Messaging System (PHIN-MS). Chief complaint data was grouped into different syndromes, including respiratory, using SQL queries and SAS 9.3. The data was then analyzed using the Early Aberration Reporting System (EARS) and compared to a previous 7-day baseline period using the cumulative sum (CUSUM) method. Aberrations representing a significant increase in cases above the threshold are flagged by the system for further review.

\section{Results}

Based on chief complaint data reported from the 22 hospitals, 96,717 respiratory illnesses were diagnosed in the emergency department from 2010 to 2012 . The number of respiratory illnesses increased each year from 22,792 in 2010 up to 47,800 in 2012. For each of the three years, aberrations for respiratory illnesses were seen during the summer months from August to September and during the cold weather months from November to January.

\section{Conclusions}

The results illustrate a seasonal increase of respiratory illness in late summer and in late fall to early winter. The increase noted in late summer may correlate to the beginning of the school year. The increase noted in late fall and early winter is consistent with seasonal influenza patterns and provides confirmation of a known seasonal trend. The analysis of syndromic surveillance data from 2010-2012 provides baseline data that could be used to help develop prevention strategies in expectation of increased respiratory illness, particularly at the onset of the school year. ${ }^{2}$ Additional research is needed to assess causes for the significant increase in respiratory illnesses from 2011 to 2012.

\section{Keywords}

Syndromic Surveillance; Seasonality; Emergency Department;
Respiratory

\section{Acknowledgments}

This work was supported by the Department of Health and Environmental Control's Division of Acute Disease Epidemiology.

\section{References}

1. van den Wijngaard CC, van Asten L, van Pelt W, and at el. Syndromic Sur veillance for Local Outbreaks of Lower-Respiratory Infections: Would it Work? PLoS One. 2010;5(4):1-6.

2. Moineddin R, Nie JX, Domb G, and et al. Seasonality of primary care utilization for respiratory diseases in Ontario: a time series analysis. BMC Health Serv Res. 2008;8:160-166.

\section{*Kelly Johnson}

E-mail: johnsok@dhec.sc.gov 